\title{
TRISOMIA 8 (SINDROME DE WARKANY 2) REPORTE DE DOS CASOS
}

\author{
Sergio Talavera Vargas Machuca1', Ismenia Gamboa Ore², Elina Mendoza Ibáñez ${ }^{3}$, María Luisa Fajardo Loo \\ Dina Torres Gonzáles ${ }^{5}$, Jackeline Zevallos Murgado ${ }^{5}$, Ruth Barrientos Marca ${ }^{5}$ Leonor Contreras Aguilar ${ }^{5}$
}

\begin{abstract}
RESUMEN
La trisomía 8 es una anomalía numérica autosómica poco frecuente entre la población, siendo la mayoría de las veces una condición letal entre individuos con líneas trisómicas puras y más tolerable en casos de mosaicismos. Esta aneuploidía se caracteriza principalmente por un déficit intelectual de moderado a severo y pobre coordinación motora. Los individuos afectados presentan malformaciones craneofaciales, donde destacan una frente prominente, paladar ojival y orejas deformadas y de implantación baja, tronco alargado, reducción de la motilidad a nivel articular, y surcos plantares profundos que es una de las principales características de esta condición, las anomalías de órganos internos tienen severidad variable. Presentamos el reporte de dos casos de trisomía 8 o síndrome de Warkany 2 nacidos en el Instituto Nacional Materno Perinatal.
\end{abstract}

Palabras clave: Trisomía 8; Síndrome de Trisomía 8; Síndrome de Warkany; Síndrome de Warkany 2 (Fuente: DeCS BIREME).

\section{TRISOMY 8 (WARKANY SYNDROME 2) REPORT OF TWO CASES}

\begin{abstract}
Trisomy 8 is an uncommon anomaly in neonates, frequently being a lethal condition among individuals with pure trisomic lines and more tolerable in mosaics cases. This aneuploidy is mainly characterized by a moderate - severe intellectual deficit and poor motor coordination. Affected individuals presents craniofacial malformations, prominent forehead, arched palate, deformed ears with low implantation, elongated trunk, reduced motility, articular defects and deep plantar grooves, which is one of the main characteristics of this condition. Abnormalities of internal organs are of varying severity. We present the report of two cases of trisomy 8 or Warkany syndrome 2 born at Instituto Nacional Materno Perinatal.
\end{abstract}

Key words: Trisomy 8; Trisomy 8 Syndrome; Warkany Syndrome; Warkany Syndrome 2 (Source:MeSH NLM).

\section{INTRODUCCIÓN}

La trisomía 8 también conocida como Síndrome de Warkany 2 es una condición genética muy poco frecuente dentro de las aneuploidías cromosómicas. Considerada una enfermedad rara debido a su incidencia muy baja, esta entidad no está relacionada a no disyunción por edad materna avanzada, y se puede encontrar dentro de la población afectada como mosaicos y menos frecuentemente como trisomías libres, siendo esta última condición la que menos sobrevida posee. Dentro de las características más saltantes de esta afección tenemos: retardo en el desarrollo psicomotor, tronco alargado y estrecho, facies dismórfica, defectos musculo esqueléticos, anomalías oculares y viscerales entre otras. Este es el segundo caso en el transcurso de siete años que conocemos del nacimiento en nuestra institución de un neonato a término con esta condición. Reportamos las características fenotípicas del propositus así como los aspectos clínicos y citogenéticos del mismo que ayuden a la valoración, el manejo clínico y seguimiento, así como una adecuada asesoría genética a los familiares. También consideramos pertinente que se considere la posibilidad de diagnóstico prenatal de esta condición por citogenética clásica y modernas técnicas moleculares.

\section{DESCRIPCIÓN DE CASOS}

\section{Caso $\mathrm{N}^{\circ} 1$}

Neonato de sexo masculino nacido a término producto de tercera gestación, de madre de 33 años, el padre de 34 años, no consanguíneos. Del primer compromiso de la madre nació un niño, aparentemente sano, del compromiso actual, la segunda gestación terminó en aborto no espontáneo.

El neonato tuvo un desarrollo gestacional normal, el parto fue por cesárea por presentar ruptura de membranas ovulares de 22 horas, con circular simple al cuello por detectado por ecografía a las 36 semanas de gestación. El volumen y aspecto de líquido amniótico fueron normales. La gestante tuvo 3 controles prenatales.

\footnotetext{
Biólogo. Docente de la Facultad de Medicina de la Universidad San Martín de Porres y de Ciencias de la Salud de la Universidad Norbert Wiener. Laboratorio de Citogenética del Instituto Nacional Materno Perinatal. Lima-Perú.

2 Bióloga. Docente de la Facultad de Medicina de la Universidad Federico Villarreal y San Martín de Porres. Laboratorio de Citogenética del Instituto Nacional Materno Perinatal. Lima-Perú.

Médico Neonatóloga Jefe del Servicio de Cuidados Intensivos del Departamento de Neonatología .

4 Médico Genetista. Instituto Nacional Materno Perinatal. Lima-Perú.

Bióloga. Laboratorio de Citogenética del Instituto Nacional Materno Perinatal. Lima-Perú
} 


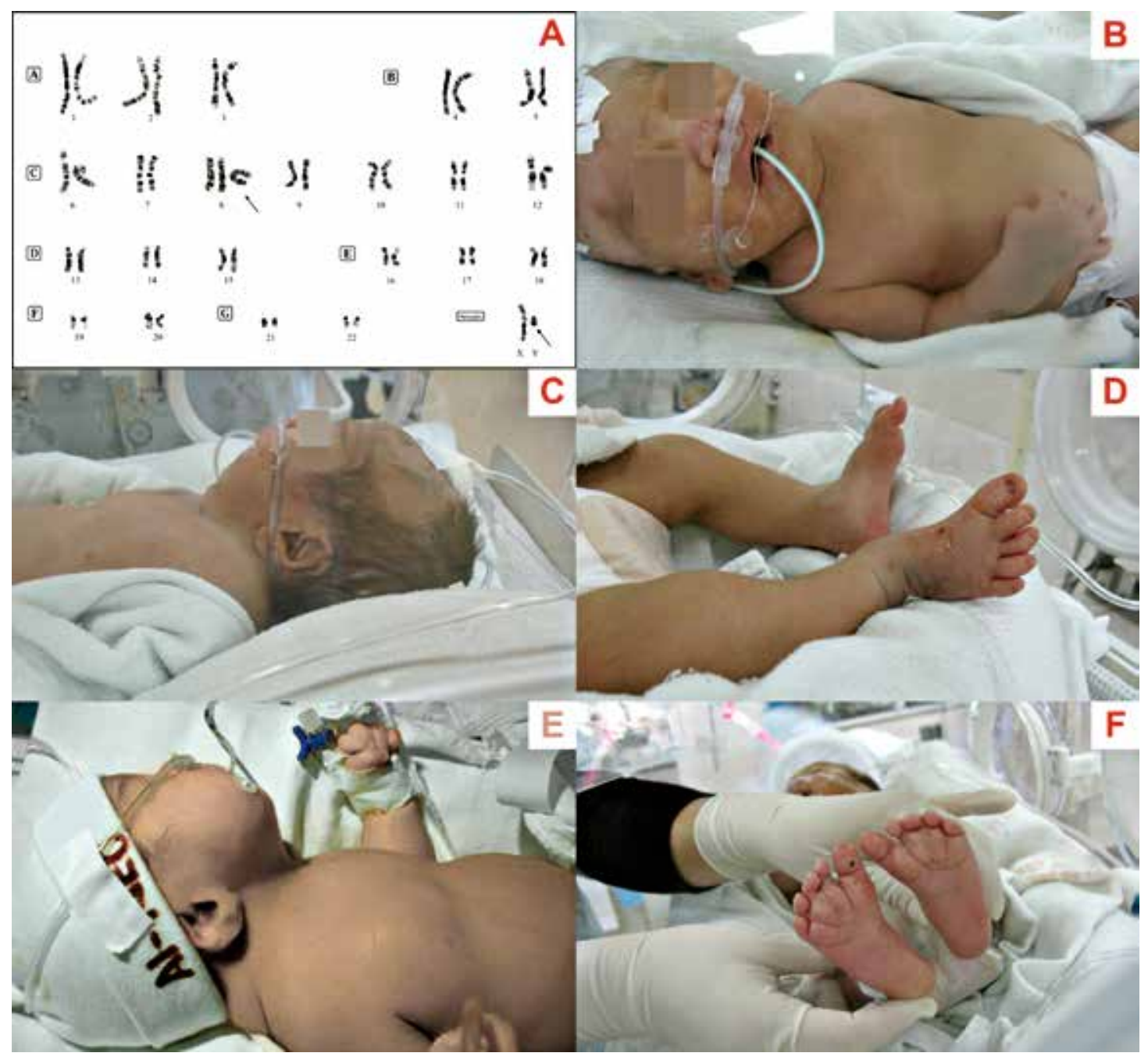

Figura 1. Caso $N^{\circ}$ 1. En A: Cariograma del neonato con una línea trisómica pura 47, $X Y,+8$. B: Características craneofaciales nótese el hipertelorismo, la nariz ancha y la boca evertida hacia abajo. C: Micrognatia y oreja displásica. D: Dorsiflexión de primer dedo de ambos pies. E: Cuello corto y alado y oreja displasica con doble lóbulo. F: Surcos plantares profundos

Nace el 05-09-09 a las 23:06 horas, por cesárea, presentación cefálica, la evaluación de Apgar fue de 3 al primer minuto y 7 a los 5 minutos, requiriendo de ventilación a presión positiva, 3,190 gr de peso, talla 50 $\mathrm{cm}$, perímetro cefálico: $35 \mathrm{~cm}$, nace a las 38 semanas de edad gestacional, siendo su peso adecuado para la edad gestacional.

Examen clínico: Rasgos dismórficos: hipertelorismo, micrognatia, pabellones auriculares displásicos y de implantaciónbaja, cuellocortocon pielredundante(pterigium colli) hipertelarquia, soplo holosistólico, clinodactilia, dorsiflexión del primer dedo del pie bilateral, hiponiquia, surcos plantares profundos longitudinales notables entre los dos primeros dedos del pie bilateral, separación hallucal criptorquidia bilateral, y ano imperforado. Evaluación neurológica: hipotonía. Por Ecografía cerebral transfontanelar: se evidencia agenesia del cuerpo calloso y colpocefalia.
La Ecocardiografía doppler indica situs ambiguous, aurícula única, Hipoplasia ventricular izquierda, canal aurículo ventricular total, atresia mitral, regurgitación tricuspídea leve, Persistencia de conducto arterioso 1.4 $\mathrm{mm}$ en boca pulmonar y de $3.8 \mathrm{~mm}$ en boca aórtica, drenaje venoso anómalo total de venas pulmonares tipo supra cardiaco a vena cava superior. El cariotipo fue $47, X Y,+8$ en 30 metafases analizadas. Fallece a los 19 días de vida. La figura 1 muestra las características fenotípicas más saltantes del Caso $\mathrm{N}^{\circ} 1$.

\section{Caso $\mathbf{N}^{\circ} 2$}

Neonato de sexo masculino, nacido pretérmino a las 36 semanas de edad gestacional, producto de quinta gestación, la madre de 33 años, cesareada anterior con un periodo intergenésico de 6 años, tuvo 7 controles prenatales, infección de tracto urinario del primer trimestre, HIV y RPR no reactivos. Ruptura prematura de membranas de 10 horas, grupo sanguíneo O+ Hemoglobina $11 \mathrm{~g} \%$, tuvo tratamiento con cefalozina y por ecografía antenatal 


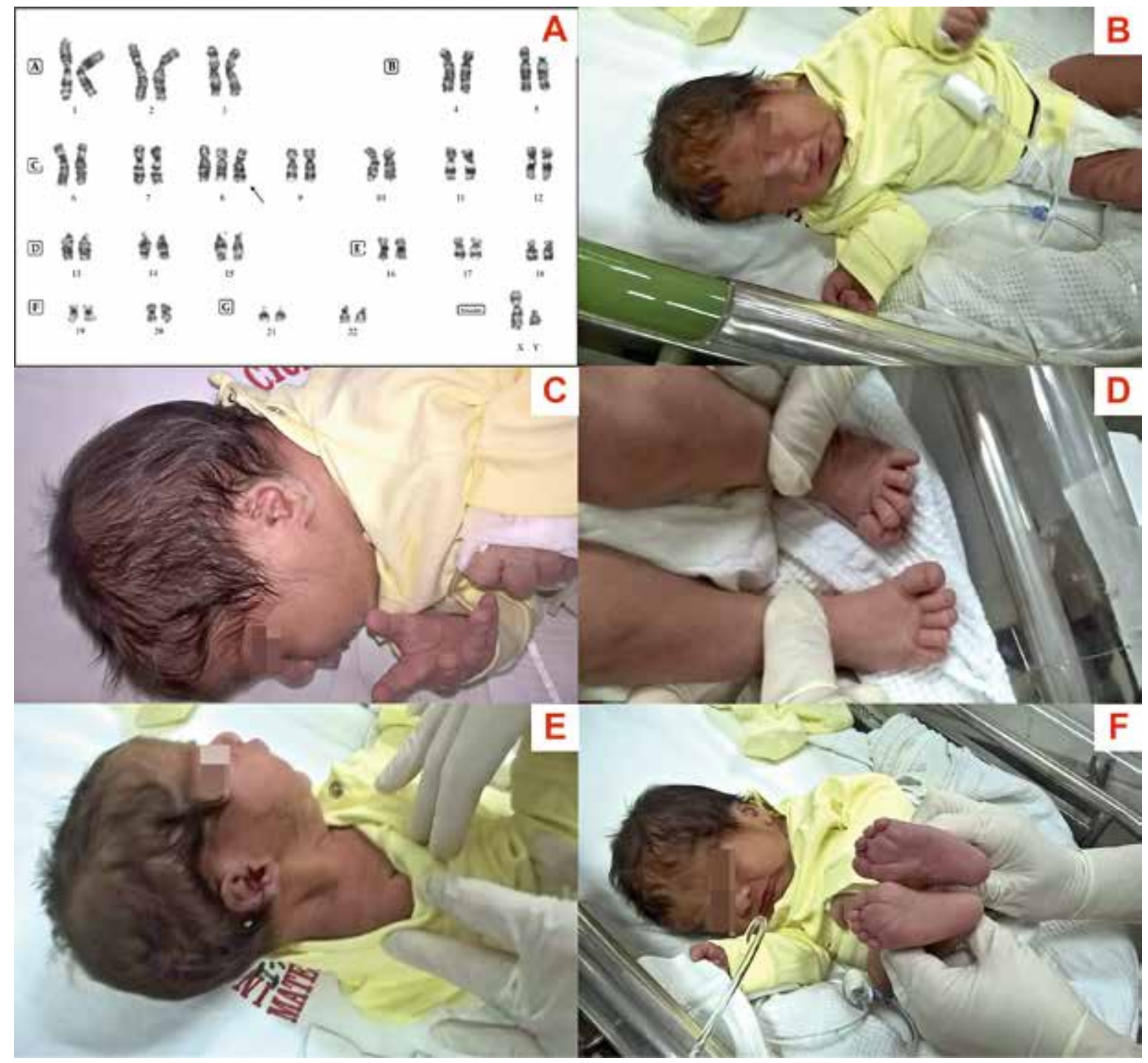

Figura 2. Caso $\mathrm{N}^{\circ}$ 2. En A: Cariograma del neonato con una línea trisómica pura 47,XY,+8. B: Características craneofaciales nótese la nariz ancha y bulbosa, hipertelorismo, ojos hundidos, prognatismo y retrognatia, la nariz ancha y la boca evertida hacia abajo. C: Pabellón auricular izquierdo deformado y lóbulo doble. D: Dedo primero dorsiflexo en ambos pies. E: Oreja derecha deformada y cuello corto y hendido. F: Surcos plantares profundos.

se evidenció una ventriculomegalia severa. El niño nace por cesárea el 23-06-16 a las 05:29 con un peso de 3,188 $\mathrm{g}$, talla $51 \mathrm{~cm}$, perímetro cefálico $36.5 \mathrm{~cm}$ con un puntaje de Apgar de 8 al primer minuto y 9 a los 5 minutos. El neonato ingresa a cuidados intermedios para estudio, ventilando espontáneamente, en el examen físico se observa cráneo con cabalgamiento de suturas, testículos no descendidos, succión no adecuada inicia endovenosa nil per os a las 35 horas de vida, pero luego succiona adecuadamente indicándose terapia orofacial, los tiempos de protrombina y de tromboplastina iniciales estuvieron alterados. Solicitada la interconsulta a Genética, a los 2 días de nacido se registra facies atípica, oreja displásica derecha, microretrognatia leve, hipertelarquia, sospecha de cromosomopatía y se indica cariotipo, el mismo día es evaluado por neuropediatría, la ecografía transfontanelar revela colpocefalia bilateral, agenesia de cuerpo calloso, hiperecogenicidad de vasos lenticuloestriados sugerentes de vasculopatía. A los 4 días se diagnostica ductus arteriosus persistente con patrón de cierre, por síntomas respiratorios se solicita examen IFI para diagnóstico de virus respiratorios por inmunofluorescencia siendo los resultados negativos. La evaluación oftalmológica al séptimo día de vida indica blefarofimosis. A los doce días es transferido para realización de resonancia magnética que revela ventriculomegalia bilateral y agenesia del cuerpo calloso, dilatación vascular venosa superior a la unión caudalotalámica derecha rama de la vena cerebral interna ipsilateral. Resonancia magnética de cerebro sin evidencia de patología inflamatoria o neoformativa. El resultado del cariotipo 47, $\mathrm{XY},+8$ (50 metafases analizadas) revela trisomía 8 pura (síndrome de Warkani 2). El niño egresa a los 19 días de vida con indicaciones de seguimiento por neuropediatría. En la figura 2 se 
muestra tanto la constitución cromosómica como las características fenotípicas más saltantes.

\section{DISCUSIÓN}

La primera referencia reportada sobre las características de esta aneuploidía es efectuada por Joseff Warkany et al en el año 1962 quien describe el caso de un niño de 6 años y medio con retardo mental moderado, pobre expresión oral, mala coordinación motora y disartria combinada con ausencia de patela y otras malformaciones congénitas, el estudio citogenético a partir de cultivos de sangre y de piel reveló una constitución cromosómica en mosaico con $70 \%$ de células anómalas $47, \mathrm{XY}$ y $22 \%$ de células normales $46, X Y$. El cariotipo concluye que el cromosoma extra podría ser un cromosoma del grupo D (grupo 13-15 con una aberración estructural, esto debido a que el cariotipo solo fue realizado con técnicas de coloración sólida o convencional ${ }^{1}$. En 1963 se reporta por primera vez un caso de trisomía del grupo C con características fenotípicas de trisomía 8². En 1968 queda establecida clínicamente la condición de trisomía C completa basándose en las características fenotípicas de tres individuos en la según el reporte de Lejeune et $\mathrm{al}^{3}$. La propuesta hecha por de Grouchy en 1971, de que el cromosoma adicional del grupo $C$ podría tratarse del cromosoma 8 , se basa en métodos de identificación por fluorescencia confirmados posteriormente por Caspersson et $\mathrm{al}^{4,5}$. La identificación citogenética por métodos de bandeo $\mathrm{C}$ y de autoradiografía por TimidinaTritio además de banda GTG en la identificación del cromosoma 8 adicional, se reporta en la publicación de Kakati en el año 1973 además de la descripción clínica de una niña con complemento cromosómico $47, \mathrm{XX},+\mathrm{C}$ con retardo psicomotor, occipucio prominente, paladar hendido, orejas de implantación baja y micrognatia, anomalías de las extremidades como clinodactilia en todos los dedos, sindactilia del segundo y tercer dedo de ambos pies y retroflexión del primer dedo en ambos pies y a nivel cardiaco defecto de septo ventricular, por cistografía se demuestra reflujo ureteral bilateral ${ }^{6}$. Lai y Gorlin establecen que el síndrome ya sea en líneas puras como en mosaicos, posee signos clínicos característicos como el retardo mental, la cabeza prominente, el paladar arqueado y deformidades de los pabellones auriculares, reducción en la motilidad articular, tronco alargado y surcos plantares profundos característicos. Sin embargo, reconocen que estas características suelen encontrarse también en otras patologías por lo que recomiendan el estudio citogenético y el establecimiento de un diagnóstico diferencial con otras condiciones como el síndrome de Fong, la artrogriposis y el síndrome oto-palato-digital ${ }^{7}$ Fineman et al, en 1975 establece que la trisomía 8 pura (T8) y la trisomía 8 en mosaico (T8m) son dos entidades diferentes pues identifica comparando sus hallazgos con los de otros 17 pacientes de una densa opacidad corneal y la deformidad clavicular como dos características solo presentes en la T8m . Gagliardi describe el caso de un individuo de 21 años, con deformidades esqueléticas a nivel de tronco (cifoescoliosis) y extremidades (malrotación articular y genu valgus) y una osteosporosis generalizada, en quien a partir de cultivo de médula ósea se encontró un complemento cromosómico de $47, \mathrm{XY},+\mathrm{C}$ identificándose el cromosoma extra como un miembro del par 8 por bandas GTG, a pesar de determinarse una T8 pura no hubo evidencias de retraso mental, lo que demuestra la gran variabilidad fenotípica existente en esta condición ${ }^{9}$.

En nuestro país esta condición fue descrita en un breve reporte clínico por de Michelena et al en el año 1992 de un niño de 23 meses de edad, con cabeza estrecha y alargada, ojos pequeños y hundidos, nariz bulbosa, puente nasal deprimido, hipertelorismo, philtrum amplio, labios delgados, y labio superior protruyente sobre el inferior, microretrognatia, orejas grandes y malformadas. El cuello corto, ancho y alado, lengua corta. El tronco amplio, hombros caídos por hipoplasia de clavículas, mamilas separadas y pelvis estrecha, displasia de huesos largos en las extremidades, incapacidad para extender los brazos. Destacan dedos de los pies mal posicionados y surcos plantares profundos, a nivel de genitales observa criptorquidia. La ecografía cardiaca indica cardiomegalia y malformación de la arteria pulmonar. La ecografía cerebral revela agenesia del cuerpo calloso y un quiste congénito en el tercer ventrículo. El cariotipo con fórmula $46, X Y / 47, X Y,+8$ del niño concluye una constitución en mosaico. $^{10}$

Las características fenotípicas y principales anomalías del desarrollo de los dos casos de neonatos nacidos en el Instituto Nacional Materno Perinatal en el transcurso de los últimos 7 años se resumen en la Tabla 1. Puede notarse que ambos casos al ser trisomías puras, comparten muchas características en común. El neonato del caso $\mathrm{N}^{\circ} 1$ fallece a los 19 días de nacido debido a la severidad de las anomalías cardiovasculares que presentó y no estuvieron presentes en el segundo caso ${ }^{18}$. El niño del caso $\mathrm{N}^{\circ} 2$ fue dado de alta a los 19 días y hasta el momento de la elaboración de este reporte permanecía con vida, una prueba más que demuestra la extrema variabilidad en la expresión fenotípica de esta condición.

Es importante realizar un estrecho seguimiento en casos de mayor sobrevida, debido a que existen características que recién se harán evidentes en la infancia y en la niñez como son el retardo en el desarrollo psicomotor. Existe una relación entre pacientes con síndrome mielodisplásico y leucemia mieloide aguda con la mutación somática de la trisomía 8 y ésta generalmente cursa con un mal pronóstico. Zollino sostiene que pacientes con un cariotipo constitucional de trisomía 8 tienen una predisposición de desarrollar cáncer y otros trastornos hematológicos ${ }^{11}$.

Siendo esta condición una enfermedad rara con una frecuencia comprendida entre 1 en 25000 y 1 en $50000^{12}$ su recurrencia es muy poco probable, sin embargo, siempre es una inquietud para los familiares de los individuos afectados conocer el origen de esta condición, 
Tabla 1. Características fenotipicas de dos neonatos con trisomia 8 (Síndrome de Warkany 2)

\begin{tabular}{|c|c|c|}
\hline Características & Caso 1 & Caso 2 \\
\hline Fecha de Nacimiento & $05 / 09 / 09$ & $23 / 06 / 16$ \\
\hline Edad materna & 33 años & 33 años \\
\hline Sexo & Masculino & Masculino \\
\hline Apgar (1 min y 5 min) & $3-7$ & $8-9$ \\
\hline Peso (g) & 3190 & 3188 \\
\hline Talla (cm) & 50 & 51 \\
\hline Perímetro cefálico (cm) & 35 & 36.5 \\
\hline Edad Gestacional & 38 & 36 \\
\hline Cariotipo & $47, X Y,+8$ & $47, X Y,+8$ \\
\hline \multicolumn{3}{|l|}{ Cabeza } \\
\hline Cabalgamiento de suturas & & + \\
\hline Hipertelorismo & + & + \\
\hline Blefarofimosis & & + \\
\hline Micrognatia & + & + \\
\hline Orejas displàsicas & + & + \\
\hline \multicolumn{3}{|l|}{ Cuello } \\
\hline Corto y redundante & + & + \\
\hline \multicolumn{3}{|l|}{ Torax } \\
\hline Hipertelarquia & + & + \\
\hline \multicolumn{3}{|l|}{ Extremidades } \\
\hline Clinodactilia & + & + \\
\hline Hiponiquia & + & + \\
\hline Dorsiflexión del primer dedo del pie & + & + \\
\hline Separación hallucal & + & \\
\hline Surcos plantares profundos & + & + \\
\hline Cardiovascular & + & \\
\hline Situs ambiguous & + & \\
\hline Aurícula única & + & \\
\hline Hipoplasia ventricular izquierda & + & \\
\hline Canal aurículo ventricular total & + & \\
\hline Atresia mitral & + & \\
\hline Regurgitación tricuspídea & + & \\
\hline Ductus arteriosus persistente & + & \\
\hline Soplo holosistólico & + & \\
\hline \multicolumn{3}{|l|}{ Sistema Nervioso Central } \\
\hline Agenesia de cuerpo calloso & + & + \\
\hline Colpocefalia & + & + \\
\hline Ventriculomegalia & & + \\
\hline Malformación vascular cerebral & & + \\
\hline \multicolumn{3}{|l|}{ Genitourinario } \\
\hline Criptorquidia bilateral & + & + \\
\hline \multicolumn{3}{|l|}{ Gastrointestinal } \\
\hline Ano imperforado & + & \\
\hline Sobrevida & 19 días & $\begin{array}{c}\text { De alta a } \\
\text { los } 19 \text { dias }\end{array}$ \\
\hline
\end{tabular}

se conocen bien los mecanismos de no disyunción, lo que falta aún, es conocer el agente o agentes que lo desencadenan. Karadima realiza un estudio molecular en trisomía 8 pura y trisomía 8 en mosaico en 26 individuos y sus progenitores utilizando 19 marcadores de DNA microsatélite y mapeando todo lo largo del cromosoma 8 , los casos estuvieron repartidos entre 20 nacidos vivos, cuatro abortos espontáneos, y dos biopsias de vellosidades coriónicas. El estudio concluye que 20 de los casos (13 maternos y 7 paternos) probablemente se deban a duplicación postcigótica debido a que se observó la reducción de homocigocidad de todos los marcadores informativos y ningún tercer alelo fue detectado. La mitad de los casos de abortos espontáneos fueron por no disyunción meiótica materna. Estos resultados contrastan con los mecanismos de no disyunción de otras trisomías autosómicas donde la mayoría de veces los errores ocurren en la meiosis materna. ${ }^{13}$

Se sabe que se manifiestan anomalías esqueléticas en la trisomía 8 , complicación que también se agudiza a mayor edad de los individuos afectados, siendo este también un motivo de seguimiento clínico. Hale y Keane ${ }^{14}$ reportan además un caso de hernia de los núcleos pulposos, a nivel espinal lumbar en un varón de 33 años con mosaicismo para la trisomía 8 , Iwatani reporta el caso de un niño afectado de trisomía 8 con situs ambiguous y hernia diafragmática además de hidronefrosis bilateral y atresia pilórica ${ }^{15}$.

Si bien es cierto, en citogenética convencional, el cariotipo realizado a partir de cultivos de linfocitos en muestras de sangre periférica, sigue siendo vigente en el diagnóstico de las anomalías numéricas y estructurales, es importante resaltar que existen métodos más modernos que facilitan la verificación de líneas puras y de mosaicos como es la hibridación in situ fluorescente (FISH) utilizando sondas centroméricas o subteloméricas específicas para el cromosoma 8, pues esta técnica permite el recuento y la discriminación visual en un mayor número de células ${ }^{17}$.

\section{CONCLUSIÓN}

La presentación de estos dos casos de trisomía 8 o síndrome de Warkany 2 en dos neonatos nacidos en el Instituto Nacional Materno Perinatal, siendo el primero de ellos producto de un trabajo presentado por Gamboa et $\mathrm{al}^{18}$, muestra algunas de las características más comunes de esta aneuploidía cromosómica como son las anomalías craneofaciales, de extremidades y malformaciones internas, compatibles con la mayoría de descripciones a nivel nacional e internacional, sugerimos la implementación de nuevas técnicas de diagnóstico molecular y de fluorescencia que permitan un mejor diagnóstico de esta entidad en la identificación de los individuos mosaicos. Es necesario un adecuado asesoramiento genético a los familiares y el seguimiento en los casos con mayor supervivencia, para un mejor manejo de sus discapacidades y mejorar de esa manera su calidad de vida.

CONFLICTO DE INTERESES. No existió conflicto de intereses entre los participantes de este reporte.

COMUNICACIONES. Sergio Hugo Talavera Vargas Machuca e-mail: stalavera35@hotmail.com 


\section{REFERENCIAS BIBLIOGRÁFICAS}

1. Warkany, J., Rubinstein, J.H., Soukup, S.W., and Curless, M.C.: Mental retardation, absence of patellae, other malformations with chromosomal mosalcism, J. PEDIAT. 61: 803, 1962

2. Jones K: Trisomy 8 syndrome. In: Smith's Recognizable Patterns of Human Malformation, edit 4. Philadelphia: W.B. Saunders, 1988; 26-29.

3. Lejeune J, Dutrillaux B, Rethoré MO, Berger R, Debray $\mathrm{H}$, Veron P, Gorce F, Grossiord A. [On three cases of C trisomy]. Ann Genet. 1969 Mar;12(1):28-35.

4. De Grouchy J, Turleau C, Leonard C: Etude en fluorescence d'une trisomie $\mathrm{C}$ mosaique, probablement 8: 46,XY/47,XY, ¿8+, Ann Genet 14:69, 1971.

5. Caspersson T, Lindsten J, Zech L, Buckton KE, Price WH. J Med Genet. 1972 Mar;9(1):1-7. Four patients with trisomy 8 identified by the fluorescence and Giemsa banding techniques.

6. Kakati S, Nihill M, Sinha AK An attempt to establish trisomy 8 syndrome. Humangenetik. 1973 Sep 20;19(3):293-300.

7. Lai CC, Gorlin RJ. Trisomy 8 syndrome Clin Orthop Relat Res. 1975 Jul-Aug;(110):238-43

8. Robert M. Fineman, Ronald C. Ablow, Rufus O. Howard, James Albright, W. Roy Breg Trisomy 8 Mosaicism Syndrome Pediatrics Volumen 56 Issue 5, 1975

9. Gagliardi AR, Tajara EH, Varella-Garcia M, Moreira LM. Trisomy 8 syndrome. J Med Genet. 1978 Feb;15(1):70-3.

10. De Michelena MI1, Sánchez R, Muñoz P, Cabello E, Rojas P, de Olazaval E. Trisomy 8: an additional case with unique manifestations [correction]. Am J Med Genet. 1992 Jul 1;43(4):697-700.

11. Zollino M, Genuardi M, Bajer J, Tornesello A, Mastrangelo S, Zampino G, Mastrangelo R, Neri G. Leuk Res. 1995 Oct;19(10):733-6.
12. Gorlin RJ, Cohen MM, Levin LS: Syndromes of the head and neck, ed 3. New York: Oxford University Press 1990:49-50.

13. Karadima G1, Bugge M, Nicolaidis P, Vassilopoulos D, Avramopoulos D, Grigoriadou M, Albrecht B, Passarge E, Annerén G, Blennow E, Clausen N, GallaVoumvouraki A, Tsezou A, Kitsiou-Tzeli S, Hahnemann JM, Hertz JM, Houge G, Kuklík M, Macek M, Lacombe D, Miller K, Moncla A, López Pajares I, Patsalis PC, Petersen $\mathrm{MB}$, et al. Origin of nondisjunction in trisomy 8 and trisomy 8 mosaicism. Eur J Hum Genet. 1998 Sep-Oct;6(5):432-8.

14. Hale NE1, Keane JF Jr. Piecing together a picture of trisomy 8 mosaicism syndrome. J Am Osteopath Assoc. 2010 Jan;110(1):21-3.

15. Iwatani S1, Takeoka E, Mizobuchi M, Yoshimoto S, Iwaki R, Bitoh Y, Nakao H. Trisomy 8 mosaicism with pyloric atresia and situs ambiguous. Pediatr Int. 2014 Dec;56(6):938-9.

16. Saumell S, Solé F, Arenillas L, Montoro J, Valcárcel D, Pedro C, Sanzo C, Luño E, Giménez T, Arnan M, Pomares $\mathrm{H}$, De Paz R, Arrizabalaga B1, Jerez A, Martínez AB, Sánchez-Castro J, Rodríguez-Gambarte JD, Raya JM, Ríos E, Rodríguez-Rivera M, Espinet B, Florensa L. Trisomy 8, a Cytogenetic Abnormality in Myelodysplastic Syndromes, Is Constitutional or Not? PLoS One. 2015 Jun 12;10(6):e0129375. 5.

17. Achandira M. Udayakumara, and Adila Al-Kindyb Constitutional trisomy 8 mosaicism syndrome: case report and review Journal of Pediatric Genetics 2 (2013) 197-201

18. Gamboa et al Trisomía 8, Síndrome de Warkany, Investigación categoría Poster Presentado en el II Congreso Internacional de Salud Materno Perinatal Auditorio del Círculo Militar, Octubre 2009 